American J. of Engineering and Applied Sciences 2 (1): 127-132, 2009

ISSN 1941-7020

(C) 2009 Science Publications

\title{
The Polishing of Cutting-Edge Polymer-on-Glass for Pigtailing Preparation
}

\author{
Mohammad Syuhaimi Ab-Rahman, Fazlinda Ab-Aziz, Noor Azie Azura Mohd Arif, \\ Saiful Dzulkefly Zan and Seri Mastura Mustaza \\ Department of Electrical, Electronic and Systems Engineering, \\ Computer and Network Security Research Group, \\ Faculty of Engineering and Built Environment, University Kebangsaan Malaysia \\ 43600 UKM Bangi, Selangor, Malaysia
}

\begin{abstract}
Problem statement: The high quality of cutting-edge surface is important in optical waveguide's efficiency. The perfect polishing of end surface is significant to deliver the best quality of light waves and minimize the device losses such as insertion loss and return loss. Hence, this research is concern on the parameters in polishing SU-8 polymer to increase the efficiency of waveguide. The main research is to study on how polishing parameter affect the cut length of the end surface of SU-8 polymer on silicon and determining the best parameters for polishing SU-8 polymer. Approach: Seven sets of rotation velocities were chosen which were 50,100, 150, 200, 250, 300 and $350 \mathrm{rpm}$ for the first part. The graph of cut length versus velocity at different sand paper size was plotted based on the data obtained from this experiment. Equation for each graph was acquired to determine relationship between these two parameters. For the second part, four samples were used. Each sample was polished with same rotation time and sandpaper size prescribed but with different rotation speed. Rotation speed is selected between 200 to $350 \mathrm{rpm}$ with rotation time of $15 \mathrm{~min}$ using sandpaper with size of $0.3 \mu \mathrm{m}$. Results: We found that the cut percentage of each rotation velocity are as follows: $50 \mathrm{rpm}: \leq 0.5 \%$, $100 \mathrm{rpm}: 0.6-1.0 \%, 150 \mathrm{rpm}: 3.8-4.8 \%, 200 \mathrm{rpm}: 7.7-10.6 \%, 250 \mathrm{rpm}: 15.7-18.3 \%$, $300 \mathrm{rpm}: 25.6-$ $27.4 \%, 350 \mathrm{rpm}: 40.0-43.7 \%$. The rotation speed suitable for polishing SU-8 polymer is below $200 \mathrm{rpm}$ and the rotation speed over the point (ex. $300 \mathrm{rpm}$ ) will cause cracking to the polymer although the silicon based layer look smooth. Conclusion: In this experiment it was found that the cut length increases as the rotation rate and the size of sand paper increased. It can also be concluded that polishing the sample at the speed of $200 \mathrm{rpm}$ is the best polishing method for polymer SU-8 waveguide, at $15 \mathrm{~min}$ rotation time with the used of $0.3 \mu \mathrm{m}$ Aluminium oxide sandpaper size. Despites giving the smooth surface, it also reduces the cutting time. The SU-8 polymer is not suitable to be polished at a high rotation speed as the surface may damage.
\end{abstract}

Key words: Waveguides polishing, SU-8 polymer, silicon substrate, rotation speed, sandpaper, length of cut

\section{INTRODUCTION}

Optical waveguide plays an important role in future high speed data transmission with speed as fast as light for transmission in vacuum medium. Nevertheless, the waveguide's efficiency is influenced by the surface structure of its side end. The best surface quality is needed for the alignment process between the fiber optic and the waveguide to increase the efficiency of fiber optic interface and waveguide. This shows that the polishing process is needed to optimize the performance and efficiency of the waveguide. A good polishing process shall optimize the performance and efficiency of the waveguide. Hence, this research is conducted to study on how the polishing parameters affect the surface structure of SU-8 polymer on silicon's side end and to find for the best polishing method in obtaining a smoother surface structure. The polishing process is a pre-process which is compulsory to align waveguide with the fiber.

The main demand of technological developments in this area is the fabrication of low-cost components for networks with high data rates ${ }^{[6]}$. Polymer waveguides have a potential of achieving the economic

Corresponding Author: Mohammad Syuhaimi Ab-Rahman, Department of Electrical, Electronic and Systems Engineering, Computer and Network Security Research Group, Faculty of Engineering and Built Environment, University Kebangsaan Malaysia, 43600 UKM Bangi, Selangor, Malaysia 